\title{
Exercise heart rate/ST segment relation in coronary artery disease
}

Sir,

Dr Elamin's paper (1982; 48: 311-20) describes a possibly accurate method of diagnosing coronary artery disease and predicting its severity in terms of the number of diseased vessels: this method is based on the calculation of the maximal ST segment/heart rate slope during exercise. In 1970 we first reported that in many patients with coronary artery disease a linear relation existed between the magnitude of exercise induced ST segment depression and indices of myocardial oxygen requirement ${ }^{1}$; we also indicated that the slope of this relation varied between patients, but since we had no angiographic data we could only speculate on the factors influencing this slope. In later studies we have shown that this relation could be modified by several therapeutic interventions. ${ }^{2-4}$

To verify Dr Elamin's data we strictly applied his methodology to 40 consecutive patients, without any previous myocardial infarction, randomly selected from our computerised data bank: from 1 January 1981 we selected the first 40 patients with (a) normal coronary angiograms $(n=10)$, (b) single vessel disease $(n=10)$, (c) double vessel disease $(n=10)$, and (d) triple vessel disease $(n=10)$. Coronary artery disease was defined as a $\geqslant 50 \%$ stenosis of a coronary vessel. All patients had a multistage maximal exercise test, and the exercise electrocardiogram (leads $\mathrm{X}, \mathrm{Y}, \mathrm{Z}$ ) was averaged and analysed by computer with a modified version of the program of the Thoraxcentrum in Rotterdam. ${ }^{5}$ The ST segment depression was measured $60 \mathrm{~ms}$ after the end of the QRS complex with the PR segment taken as the zero reference; the heart rate was measured simultaneously and the maximal ST/HR slope calculated. The results of this preliminary analysis are shown in the Table.

Our data show that there was an overlap between the ST/HR slopes measured in patients with and without coronary artery disease. The mean ST/HR slope was, however, significantly less in patients without disease $(p<0.05$ vs single vessel disease; $p<0.001$ $v s$ double or triple vessel disease). More importantly, the ST/HR slopes were not significantly influenced by the number of diseased vessels, and the severity of the disease could therefore not be predicted from the value of the ST/HR slope.
Table Results of preliminary analysis

\begin{tabular}{lcc}
\hline Angiographic data & \multicolumn{2}{c}{ Maximal ST/HR slope } \\
\cline { 2 - 3 } & Mean $\pm S D$ & Range \\
\hline No disease & $12 \cdot 4 \pm 11 \cdot 7$ & $-21,+30$ \\
Single vessel disease & $41 \cdot 8 \pm 40 \cdot 8$ & $-7,+110$ \\
Double vessel disease & $42 \cdot 0 \pm 23 \cdot 9$ & $+13,+81$ \\
Triple vessel disease & $50 \cdot 2 \pm 36 \cdot 6$ & $+24,+142$ \\
\hline
\end{tabular}

Although we recognise that our methodology differs slightly from that of Elamin et al, these minor differences cannot explain the total disagreement between our findings. The data as well as the concepts presented by Dr Elamin et al should thus be considered with caution, and we value Fox's wise editorial. $^{6}$

J M Detry, R Vanbutsele, Division of Cardiology,

Saint-Luc University Hospital, B-1200 Brussels,

Belgium.

\section{References}

1 Detry JMR, Piette F, Brasseur LA. Hemodynamic determinants of exercise ST-segment depression in coronary patients. Circulation 1970; 42: 593-9.

2 Detry JMR, Bruce RA. Effects of nitroglycerin on "maximal" oxygen intake and exercise electrocardiogram in coronary heart disease. Circulation 1971; 43: 155-63.

3 Detry JMR, Bruce RA. Effects of physical training on exertional ST-segment depression in coronary heart disease. Circulation 1971; 44: 390-6.

4 Rousseau MF, Brasseur LA, Detry JM. Haemodynamic and electrocardiographic effects of Practolol during upright exercise in coronary heart disease. Cardiovasc Res 1973; 7: 306-12.

5 Simoons ML, Hugenholtz PG. Estimation of the probability of exercise-induced ischemia by quantitative ECG analysis. Circulation 1977; 56: 552-9.

6 Fox KM. Exercise heart rate/ST segment relation: perfect predictor of coronary disease? [Editorial]. $\mathrm{Br} \mathrm{Heart} \mathcal{F}$ 1982; 48: 309-10. 
This letter was shown to the authors, on whose behalf Professor Linden and Dr Mary reply below:

Sir,

The above letter contains the second claim that if different exercise tests are completed then it is possible to obtain different results. The first claim that our test was inadequate was made by Ilsley et al.$^{1}$ They used a test which was different from ours in that lead CM5 was omitted; it is a matter of fact that if we omit the lead CM5 from our results from 230 patients then we get the same results as Ilsley et al ${ }^{1}$ (see Table). Moreover, Ilsley et $a^{1}$ obtained their results retrospectively from their data bank, and the test was not performed as particularly described by us.

Similar criticisms can be made of the test described in the letter above, which again claims to show different results. Firstly, again lead CM5 was excluded-in fact, instead of a 13 lead system, as used by us, a three lead system $(\mathrm{X}, \mathrm{Y}, \mathrm{Z})$ was used. Although such a three lead system should not give vastly different results on analysis, Macfarlane et al have pointed out from a systematic comparison that these two systems provide different information. ${ }^{2}$ Secondly, the protocols were different. Thirdly, the ST segment depression was measured at $60 \mathrm{~ms}$ after the end of the QRS complex not at $80 \mathrm{~ms}$. Fourthly, coronary artery disease was defined as $50 \%$ or more constriction instead of $75 \%$ or more. No comment is made in their letter as to whether measurements were made in the steady state or whether incremental workload steps were tailored for each patient. It is difficult to believe that their words "slightly different" have any real meaning.
Although there is much we disagree with in Fox's editorial ${ }^{3}$ it contained a wise commment that "we had better wait and see," implying that others should attempt to repeat our trial. We would hope, however, that the repeated trials would at least involve exact repetition of our test. It would be a tragedy if a potentially reasonable non-invasive method of helping to diagnose this very important disease was rejected because of technical differences.

R J Linden, D A S G Mary,

Department of Cardiovascular Studies, University of Leeds,

Leeds.

\section{References}

1 Ilsley C, Canepa-Anson R, Rickards A. Maximal ST/ heart rate slope: a reliable predictor of severity of coronary artery disease? [Abstract] Br Heart f 1983; 49: 290.

2 Macfarlane PW, Melville DI, Horton MR, Bailey JJ. Comparative evaluation of the IBM (12-lead) and Royal Infirmary (orthogonal three-lead) ECG computer programs. Circulation 1981; 63: 354-9.

3 Fox KM. Exercise heart rate/ST segment relation: perfect predictor of coronary disease? [Editorial]. Br Heart $\mathrm{F}$ 1982; 48: 309-10.

Table Comparative results

\begin{tabular}{llllll}
\hline & \multicolumn{2}{l}{ Leeds test } & & \multicolumn{2}{l}{ Test excluding lead CM5 } \\
\cline { 2 - 3 } & No of patients & Success $(\%)$ & & No of patients & Success (\%) \\
\hline No significant disease & 38 & 100 & & 38 & 100 \\
Single vessel disease & 56 & 100 & & 50 & 89 \\
Double vessel disease & 85 & 100 & & 78 & 92 \\
Triple vessel disease & 51 & 100 & & 205 & 76 \\
Total & 230 & 100 & & & 89 \\
\hline
\end{tabular}

